\title{
Overexpression of cysteine cathepsin $L$ is a marker of invasion and metastasis in ovarian cancer
}

\author{
WEI ZHANG $^{1}$, SUMEI WANG ${ }^{1}$, QI WANG ${ }^{1}$, ZHIJUN YANG $^{1}$, ZHONGMIAN PAN $^{1}$ and LI LI $^{1,2}$ \\ ${ }^{1}$ Department of Gynecologic Oncology, Affiliated Tumor Hospital of Guangxi Medical University; \\ ${ }^{2}$ Medical Scientific Research Centre, Guangxi Medical University, Nanning, Guangxi 530021, P.R. China
}

Received October 8, 2013; Accepted November 29, 2013

DOI: 10.3892/or.2014.2967

\begin{abstract}
Cysteine cathepsins (CTSs) are involved in the degradation and remodeling of the extracellular matrix and are associated with cellular transformation, differentiation, motility and adhesion in cancer development. Previous studies indicate that CTSs may be involved in ovarian cancer invasion and metastasis. However, due to the lack of large sample clinical studies and direct experimental evidence for the relationship between the expression of CTSs and invasion and metastasis, the diagnostic and prognostic value of CTSs in ovarian cancer progression has not been elucidated. In the present study, we observed that expression levels of CTSB, CTSL and CC in malignant ovarian tumors were significantly higher than the expression levels in benign tumors and normal ovarian tissues, yet their associations with clinicopathological features varied. In particular, CTSL was related to lymph node metastasis, CC was related to liver metastasis and omental metastasis, and CTSB and CTSL expression levels were found to be independent prognostic factors in ovarian cancer. Further study indicated that the serum level of CTSL was significantly higher in patients with ovarian malignant tumors than the levels in benign tumors and healthy controls, and the levels were elevated in low grade and advanced stage compared to the levels in high grade and early stage disease, suggesting that the serum level of CTSL may be a useful serum marker for the diagnosis of ovarian cancer. Furthermore, the expression of CTSL in ovarian cancer cells can greatly enhance the ability of cell invasion and metastasis, although no change was observed for cell adhesion. Taken together, we demonstrated that the overexpression of CTSL is involved in tumor invasion and metastasis, and the CTSL level in serum may be a marker for invasion and metastasis in ovarian cancer.
\end{abstract}

Correspondence to: Professor Li Li, Department of Gynecologic Oncology, Affiliated Tumor Hospital of Guangxi Medical University, 71 Hedi Road, Nanning, Guangxi 530021, P.R. China E-mail: lili@gxmu.edu.cn

Key words: cysteine cathepsins, ovarian cancer, tumor metastasis, biological behavior, clinical significance

\section{Introduction}

Ovarian cancer has the highest mortality rate of all gynecological cancers and is the fifth leading cause of death among women (1). Approximately $90 \%$ of human ovarian cancers are thought to originate from the ovarian surface epithelium (2). Ovarian cancer is difficult to diagnose at an early stage due to the lack of specific symptom and physical signs, and ovarian cancer has a high rate of metastasis in the early stage. Approximately $70 \%$ of the patients are diagnosed with FIGO stage III or IV, with a poor 5-year survival rate. Although the ideal primary cytoreductive surgery and combination chemotherapy with platinum have improved the prognosis of patients with advanced ovarian cancer, the 5-year survival rate remains $\sim 40 \%(3,4)$. Cysteine cathepsins (CTSs) are a family of cysteine proteases which function primarily in protein degradation in the lysosomes in the majority of cell types (5). CTSs are involved in the degradation and remodeling of the extracellular matrix and are associated with cellular transformation, differentiation, motility and adhesion. These functions are also related to cancer cell invasion and metastasis. CTSs are believed to play important roles in ovarian cancer invasion and metastasis. Athanassiadou et al (6) revealed that cathepsin D (CTSD) is an indicator of malignancy in serous ovarian carcinoma, as its expression is higher in serous ovarian carcinoma than in benign serous ovarian tumors. In addition, Nishida et al (7) observed significantly increased serum levels of CTSL in patients with ovarian cancer $(\mathrm{P}<0.05)$. Moreover, ovarian cancer samples were found to express higher levels of CTSL mRNA than those of uterine cancer, benign ovarian tumors, and normal ovarian tissue samples. Kolwijck et al (8) found that the ratio of $\mathrm{CysC} / \mathrm{CatB}$ was significantly lower in patients with metastasis compared with this ratio in localized epithelial ovarian cancer $(\mathrm{EOC})(\mathrm{P}=0.025)$. The ratios of $\mathrm{CysC} / \mathrm{CatH}$ and $\mathrm{CysC} / \mathrm{CatX}$ differed significantly between histological subtypes $(\mathrm{P}=0.012$ and $\mathrm{P}=0.035$, respectively) and were significantly higher in high-grade tumors when compared with the ratios in low-grade tumors $(\mathrm{P}=0.031$ and $\mathrm{P}=0.039$, respectively). Neither cathepsins nor their ratios were significant predictors of survival for EOC patients. Meanwhile, analogical study results have been reported in other cancer types. Therefore, CTSs are considered to be potential prognostic factors for the aggressiveness of ovarian cancer, and may contribute to the invasion of ovarian cancer cells (9). 
Disappointingly, however, due to the lack of large sample clinical studies and directly experimental evidence for the relationship between the expression of CTSs and invasion and metastasis, the scientific community has not reached a general consensus on the diagnostic and prognostic value of CTSs in ovarian cancer progression. In the present study, based on the analysis of the relationship between the expression of CTSB, CTSL, CTSD and CC in ovarian epithelial carcinoma tissues and the CTSL concentration in the serum of patients with ovarian epithelial carcinoma, we aimed to explain whether CTSs may act as clinicopathological factors, and whether the overexpression of CTSL promotes cell invasion and metastasis in ovarian cancer cells.

\section{Materials and methods}

\section{Samples}

Tissue samples. All tissue samples were obtained from patients who underwent surgery at the Department of Gynecologic Oncology, Affiliated Tumor Hospital of Guangxi Medical University, Nanning, Guangxi from October 2002 to October 2009, and diagnoses were confirmed by a pathologist. This research included 47 epithelial malignant ovarian tumors (24 serous, 12 mucinous and 11 undifferentiated), 20 benign ovarian tumors (12 serous and 8 mucinous) and 21 normal ovarian tissues (obtained from patients with hysteromyoma who received hysterectomy + hapl-oophorotomy). In the malignant group, the median age of the patients was 45.38 years (range, 34-73), and 20 patients had stage I-II tumors, and 27 patients had stage III-IV tumors according to the International Federation of Gynecology and Obstetrics (FIGO) classification, and 17 had high and intermediate degrees of differentiation and 20 had poor differentiation. All of the patients were followed up (100\%). The survival of the patients ranged from 8 to 67 months, and the median survival time was 29.81 months. The 3 -year survival rate was $49 \%$ and the 5-year survival rate was $32 \%$. In the benign group, the median age of the patients was 40.6 years (range, 24-68), and in the normal group, the median age of the individuals was 44.8 years (range, 42-53). All tissue specimens were collected from the primary tumor lesion during surgery. A portion of each specimen was sent for histopathological examination, and the remaining portion was immediately stored in a liquid nitrogen tank ready for RNA isolation.

Human serum samples. Serum samples were obtained from patients who underwent surgery at the Department of Gynecologic Oncology, Affiliated Tumor Hospital of Guangxi Medical University, Nanning, Guangxi. This research included 177 epithelial malignant ovarian tumors (109 serous, 54 mucinous and 14 undifferentiated) and 100 benign tumors (62 serous, 24 mucinous and 14 benign teratoma). Among the patients with malignant tumors, 83 patients had stage I-II tumors and 134 patients had stage III-IV tumors according to FIGO classification. The median age of the patients with malignant tumors was 44.6 years (range, 16-67), and the median age of the patients with benign tumors was 35.6 years (range, 14-64). Serum samples of normal controls were obtained from 101 healthy females undergoing routine physical examinations.
Table I. Specific primers of the genes.

\begin{tabular}{llc} 
Gene & \multicolumn{1}{c}{ Primer sequence } & $\begin{array}{c}\text { Fragment } \\
\text { size (bp) }\end{array}$ \\
\hline CTSB & $\begin{array}{l}\text { F: 5'-CAGATTGCCTCCTTATGAC-3' } \\
\text { R: 5'-GAGAAGTTAAGATGAAGTCCC-3' }\end{array}$ & 328 \\
CTSL & F: 5'-ATACAGGGAAGGGAAAC-3' & 494 \\
& R: 5'-TAGGGATGTCCACAAAG-3' & \\
CTSD & F: 5'-GCTCTGTGGAGGACCTGATTG-3' & 378 \\
& R: 5'-AGGCTGACGACGCTGACTG-3' & \\
CC & F: 5'-AACATAGCCAGCTACGAC-3' & 456 \\
& R: 5'-GCAAGTAGGATGGAGTGAG-3 & \\
GAPDH & F: 5'-GAAGGTGAAGGTCGGAGT-3' & 225 \\
& R: 5'-GAAGATGGTGATGGGATTTC-3' & \\
\hline
\end{tabular}

F, forward; R, reverse.

The study was endorsed by the Ethics Committee of the Guangxi Medical University. All subjects received an explanation of the aims of the study and signed informed consent. All subjects understood that they could withdraw from the study at any time without influencing their oncological or general medical treatment.

RT-PCR analysis. Total RNA was extracted from frozen tissues by TRIzol reagent (Invitrogen, Carlsbad, CA, USA), and cDNAs were synthesized using AccuPower RT PreMix (Invitrogen). The cDNA was serially diluted 10-fold and quantitatively equalized for PCR amplification using specific primers (Table I). The PCR amplification was performed under the following conditions: initial denaturation at $94^{\circ} \mathrm{C}$ for 5 min, followed by a variable number of 35 cycles: $94^{\circ} \mathrm{C}$ for $30 \mathrm{sec}$, specific annealing temperature for $30 \mathrm{sec}$, elongation at $72^{\circ} \mathrm{C}$ for $45 \mathrm{sec}$; and a final elongation at $72^{\circ} \mathrm{C}$ for $5 \mathrm{~min}$. The PCR products were visualized on $1.5 \%$ agarose gels containing ethidium bromide. GAPDH was used as a control. The ratio of the grayscale value of the gene to the value of GAPDH was determined as the relative expression level of the gene.

Enzyme-linked immunosorbent assay (ELISA) detection. Two microliters of peripheral blood was obtained from patients prior to any treatment. Sera were collected and stored at $-80^{\circ} \mathrm{C}$. ELISA for CTSL was performed using an immunoassay kit (Boatman Biotech, Shanghai, China) according to the manufacturer's instructions. Goat polyclonal antibody against CTSL and standard substance were purchased from Santa Cruz Biotechnology (Santa Cruz, CA, USA). The optical density (OD) at 450 was determined. The standard curve was established by the value of OD450 vs. the concentration of the standard substance. The level of protein was calculated in accordance with standard curve, and the equation of the standard curve for CTSL was $y=a\left(1-e^{-b x}\right)$, where $a=1,096.1137$, $\mathrm{b}=0.0416$ and $\mathrm{r}=0.8578$.

Construction of the pcDNA3.1-CTSL eukaryotic expression plasmid. The construction of the pcDNA3.1-CTSL expression 
plasmid was performed as follows. Briefly, the primer was designed according to the cDNA sequence of CTSL which was deposited in the GenBank database, for which the restriction sites of XhoI and BamHI were inserted into both ends of the CTSL open reading frame. The specific primers were upstream primer, 5'-GCCTCGAGCATGAATCCTACACTCA TCCTTG-3' and downstream primer, 5'-GCAGGATCCTC ACACAGTGGGGTAGC-3'. The purified PCR product of CTSL was linked with the pMD18-T vector using T4 DNA ligase (Takara Co), and the constructed pMD18-T-CTSL plasmid was confirmed by sequencing. Then the pcDNA3.1 and pMD18-T-CTSL vectors both digested with BamHI and $X h o I$ were purified and linked to develop recombinant pcDNA3.1-CTSL. The pcDNA3.1-CTSL DNA was confirmed by sequencing.

Construction of the CTSL-siRNA expression vector. Four siRNA primers for CTSL and the primers for the control genes were designed as follows: i) CTSL-441, GGCGATGCAC AACAGATTA; ii) CTSL-906, GTATGTTCAGGATAATG GA; iii) CTSL-1202, GCACAGAATCAGATAACAA; iv) CTSL-1265, CGGATTTGAAAGCACAGAA; v) NC, TTCT CCGAACGTGTCACGT; vi) GAPDH, GUAUGACAACAG CCUCAAGTT. The target labeled with fluorescence was transfected into A2780 cells. Total RNA was extracted by TRIzol reagent, and cDNAs were synthesized using AccuPower RT PreMix. The expression of CTSL mRNA in cells transfected with the target and the control was measured by PCR. The gel imaging system was used to analyze the grayscale ratio of CTSL vs. $\beta$-actin, and the best siRNA silencing efficiency was determined according to the grayscale.

In accordance with the requirement of the expression plasmid pSilencer ${ }^{\mathrm{TM}} 4.1-\mathrm{CMV}$-neo (Ambion Co.), the sequence TTCAAGAGA was selected as the loop. DNA sequence loop ends are complementary to the siRNA target sequence. The DNA sequence of short hairpin RNA (small hairpin RNAs, shRNA) with BamH1 and HindIII sticky ends was designed. At the same time, a non-human short hairpin RNA sequence was designed as a negative control. The oligonucleotide sequences for CTSL were 5'-GATCCGCACAGAATCAG ATAACAATTCAAGAGATTGTTATCTGATTCTGTGCA GA-3' and 5'-AGCTTCTGCACAGAATCAGATAACAATC TCTTGAATGTTATCTGATTCTGTGCG-3', and the oligonucleotide sequences for the control were 5'-GATCCCCGCG AACGAAATAAAATATTCAAGAGATATTTTATTTCGT TCGCGGAGA-3' and 5'-AGCTTCTCCGCGAACGAAATA AAATATCTCTTGAATATTTTATTTCGTTCGCGGG-3'.

The oligonucleotide was annealed to form a pair of oligonucleotides, then $\mathrm{pSilencer}{ }^{\mathrm{TM}} 4.1$-neo vector was digested and linearization. The oligonucleotide pairs were connected with the linearization vector in a 2:1 ratio by T4 DNA ligase, and transformed into DH5a competent cells. The plasmid was extracted using a plasmid extraction kit (Promega) and confirmed by sequencing, and named as recombinant plasmids pSilencer ${ }^{\mathrm{TM}} 4.1-\mathrm{CTSL}$ and $\mathrm{pSilencer}{ }^{\mathrm{TM}} 4.1-$ Control, respectively.

Transfection of HO8910 and A2780 cells with plasmid DNA. The plasmid DNA of pcDNA3.1-CTSL and pcDNA3.1 were transfected into HO8910 cells using liposome
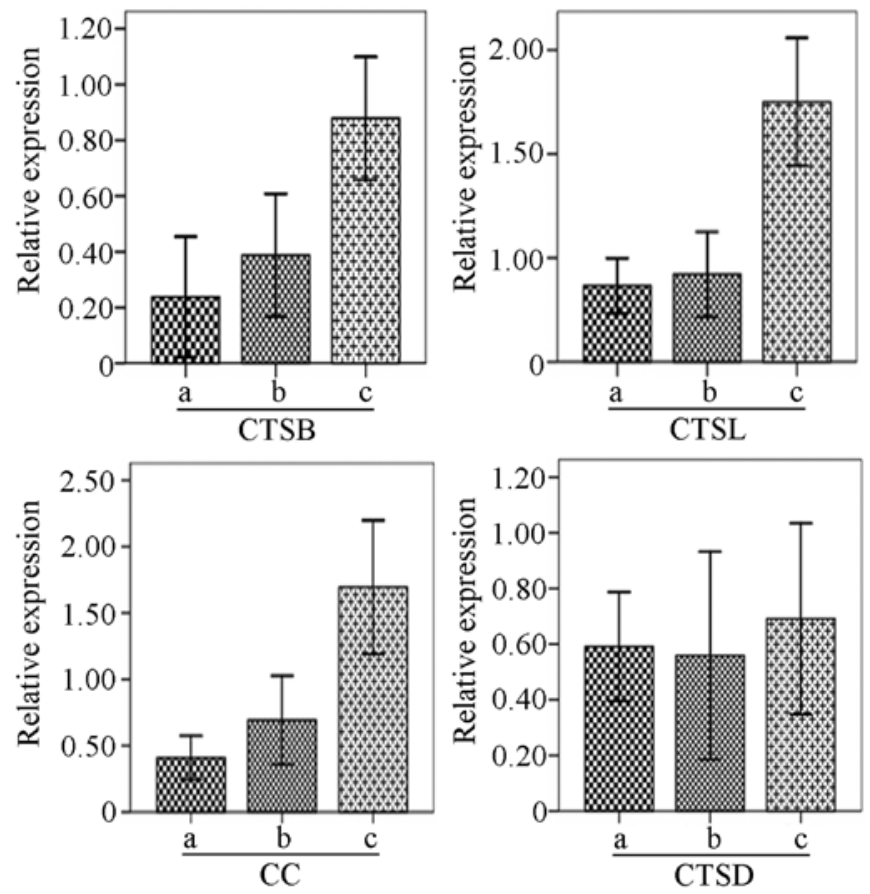

Figure 1. mRNA expression of CTSB, CTSL, CC and CTSD in (a) normal, (b) benign and (c) malignant ovarian tissues.

Lipofectamine 2000 reagent (Invitrogen), and the plasmid DNA of pSilencer ${ }^{\mathrm{TM}} 4.1$-CTSL, pSilencer ${ }^{\mathrm{TM}} 4$.1-Control and pSilencer ${ }^{\mathrm{TM}} 4.1$ was transfected into A2780 cells. G418 reagent was used for the selection of the transfected cells. The CTSL mRNA and protein expression in each subgroup of cells was measured using RT-PCR and western blotting. The transfected cells were named HO8910-CTSL, HO8910-pcDNA3.1, A2780-CTSL, A2780-Control and A2780-pSilencer, respectively.

Methods to determine the cell biological behavior. Cell growth was measured using the MTT assay, cell cycle was determined by flow cytometric assay, and DNA content and the cell number and cell proportion in G1, G2, S phases of the cell cycle were analyzed by MultiCycle software. Cell invasion in vitro was measured by Matrigel invasion assay, and cell migration in vitro was measured by Transwell migration assay.

Data analysis. SPSS 10.0 statistical software was used for data analysis. $\mathrm{P}<0.05$ was considered to indicate a statistically significance result.

\section{Results}

mRNA expression of CTSB, CTSL, CC and CTSD, and their associations with clinicopathological features and prognosis in ovarian cancer. As shown in Fig. 1, the mRNA expression of CTSB, CTSL and CC in malignant ovarian tissues was higher than the expression in the normal and benign tissues $(\mathrm{P}<0.01)$, while no significant difference in expression was observed between the normal and benign ovarian tissues $(\mathrm{P}>0.05)$. In addition, the ratio of CTSB expression vs. CC expression in benign and malignant tissues was higher than the ratio in 
Table II. Relationship betweem CTS expression and the clinicopathological factors in epithelial ovarian cancer (mean \pm SD).

\begin{tabular}{|c|c|c|c|c|c|}
\hline Clinicopathological factors & $\mathrm{n}$ & CTSB & CTSL & $\mathrm{CC}$ & CTSD \\
\hline Epithelial ovarian cancer & 47 & & & & \\
\hline Serous cystadenocarcinoma & 21 & $0.763 \pm 0.756$ & $1.416 \pm 1.202$ & $1.149 \pm 0.667$ & $1.122 \pm 1.120$ \\
\hline Mucinous cystadenocarcinoma & 9 & $0.191 \pm 0.244^{\mathrm{b}}$ & $1.324 \pm 1.241$ & $1.143 \pm 1.521$ & $0.583 \pm 0.942$ \\
\hline Poorly differentiated adenocarcinoma & 17 & $0.6977 \pm 0.504$ & $1.143 \pm 1.090$ & $2.661 \pm 2.60$ & $1.4062 \pm 1.9094$ \\
\hline \multicolumn{6}{|l|}{ Stage } \\
\hline I-II stage & 12 & $0.437 \pm 0.320$ & $0.632 \pm 0.889$ & $1.317 \pm 0.897$ & $1.101 \pm 1.368$ \\
\hline III-IV stage & 35 & $0.690 \pm 0.618$ & $1.460 \pm 1.068$ & $1.824 \pm 1.910$ & $1.027 \pm 1.253$ \\
\hline \multicolumn{6}{|l|}{ Pathological grade ${ }^{a}$} \\
\hline G1-G2 & 14 & $0.68 \pm 0.783$ & $0.748 \pm 1.011$ & $1.092 \pm 0.646$ & $0.457 \pm 0.665$ \\
\hline G3 & 23 & $0.674 \pm 0.475$ & $1.54 \pm 1.103$ & $2.060 \pm 2.138$ & $0.917 \pm 1.462$ \\
\hline \multicolumn{6}{|l|}{ Liver metastases } \\
\hline No & 39 & $0.628 \pm 0.606$ & $1.12 \pm 1.08$ & $1.092 \pm 0.646$ & $0523 \pm 0.719$ \\
\hline Yes & 8 & $0.608 \pm 0.298$ & $1.86 \pm 0.839$ & $2.895 \pm 2.367$ & $1.504 \pm 2.299$ \\
\hline \multicolumn{6}{|l|}{ Lymph node metastasis } \\
\hline No & 35 & $0.569 \pm 0.469$ & $1.07 \pm 1.14$ & $1.441 \pm 1.159$ & $0.690 \pm 1.326$ \\
\hline Yes & 12 & $0.766 \pm 0.771$ & $1.67 \pm 0.629$ & $2.292 \pm 2.557$ & $0.691 \pm 0.722$ \\
\hline \multicolumn{6}{|l|}{ Omentum metastasis } \\
\hline No & 20 & $0.648 \pm 0.702$ & $1.25 \pm 1.105$ & $1.230 \pm 0.947$ & $0.707 \pm 1.495$ \\
\hline Yes & 27 & $0.602 \pm 0.417$ & $1.24 \pm 1.08$ & $2.081 \pm 2.088$ & $0.678 \pm 0.889$ \\
\hline \multicolumn{6}{|l|}{ Ascites (ml) } \\
\hline$<500$ & 26 & $0.6835 \pm 0.711$ & $1.171 \pm 1.174$ & $1.203 \pm 1.052$ & $0.347 \pm 0.493$ \\
\hline$>500$ & 21 & $0.540 \pm 0.243^{\mathrm{a}}$ & $1.345 \pm 0.969$ & $2.255 \pm 2.241$ & $1.115 \pm 1.582$ \\
\hline \multicolumn{6}{|l|}{ Residual tumor (cm) } \\
\hline$<2$ & 35 & $0.690 \pm 0.588$ & $1.27 \pm 1.16$ & $1.655 \pm 1.478$ & $0.753 \pm 1.310$ \\
\hline$>2$ & 12 & $0.432 \pm 0.472$ & $1.16 \pm 0.84$ & $1.810 \pm 2.048$ & $0.508 \pm 0.610$ \\
\hline
\end{tabular}

${ }^{a}$ Pathological classification refers only to ovarian epithelial carcinoma; ${ }^{\mathrm{b}}$ serous ovarian carcinoma and mucinous carcinoma.

the normal controls $(\mathrm{P}<0.05)$, but no difference was detected between the ratio in the benign and malignant tissues.

Correlation between the expression levels of CTSB, CTSL, $\mathrm{CC}$ and CTSD and clinicopathological features in the ovarian malignancies was varied (Table II). The mRNA expression of CTSB had no relationship with surgical pathological stage, histological grade, lymph node metastasis, and residual tumor in the malignant ovarian tumors $(\mathrm{P}>0.05)$, while the CTSB expression in patients with ascites $>500 \mathrm{ml}$ was significantly higher than the expression in patients with ascites $<500 \mathrm{ml}$ $(\mathrm{P}=0.006)$. In addition, the CTSB expression in serous carcinoma was higher than the expression in mucinous carcinoma $(\mathrm{P}=0.047)$. The CTSL expression had a weaker association with histological type, residual tumor, liver metastasis, omental metastases and ascites $(\mathrm{P}>0.05)$, while its expression in stage III-IV ovarian malignancies was significantly higher than the expression in stage I-II tumors $(\mathrm{P}=0.02)$. Moreover, the CTSL expression in the highly differentiated malignant ovarian tumors was significantly higher than that in the poorly differentiated tumors $(\mathrm{P}=0.041)$, and the expression in ovarian malignancies with lymph node metastasis was significantly higher than the expression in patients without lymph node metastasis $(\mathrm{P}=0.026)$. The $\mathrm{CC}$ expression in malignant ovarian tumors had a limited relationship with histological type, surgical stage, lymph node metastasis and the residual tumor $(\mathrm{P}>0.05)$. However, the $\mathrm{CC}$ expression in patients with poorly differentiated adenocarcinoma, or with liver metastasis and omentum metastasis, or with ascites volume $>500 \mathrm{ml}$ was significantly higher than the expression in patients with moderately differentiated adenocarcinomas $(\mathrm{P}=0.016)$, or without metastasis $(\mathrm{P}=0.027)$ or with the amount of ascites $<500 \mathrm{ml}(\mathrm{P}=0.039)$, respectively. The expression of CTSD had weak associations with surgical stage, pathological type, histological grade, lymph node metastasis and residual tumor $(\mathrm{P}>0.05)$, while its expression in patients with liver metastasis was significantly higher than the expression in patients without liver metastasis $(\mathrm{P}=0.029)$, and the expression in patients with ascites $>500 \mathrm{ml}$ was higher than the expression in patients with ascites $<500 \mathrm{ml}(\mathrm{P}=0.024)$.

The associations of the expression of the CTS genes with prognosis in patients with ovarian cancer were analyzed using Kaplan-Meier survival curve and long-rank testing. As shown in Fig. 2, the median survival time of the patients with tumors exhibiting negative CTSB expression was longer than that of the patients with CTSB-positive expression in the tumors, but the difference was not statistically significant. The survival 
Table III. Results of Cox Proportional-Hazards Regression.

$95 \% \mathrm{CI}$

\begin{tabular}{lccccccrr}
\cline { 5 - 8 } Clinicopathological factors & B & SE & Wald & df & Sig & Exp (B) & Lower & Upper \\
\hline Tumor stage & 0.360 & 0.735 & 0.240 & 1 & 0.625 & 1.433 & 0.339 & 6.053 \\
Tumor type & -1.292 & 0.930 & 1.931 & 1 & 0.165 & 0.275 & 0.044 & 1.700 \\
Tumor grade & 0.407 & 0.378 & 1.164 & 1 & 0.281 & 1.503 & 0.717 & 3.150 \\
Liver metastasis & -1.687 & 1.312 & 1.652 & 1 & 0.199 & 0.185 & 0.014 & 2.424 \\
Omentum metastasis & 1.756 & 1.087 & 2.607 & 1 & 0.106 & 5.789 & 0.687 & 48.778 \\
Lymph node metastasis & -0.616 & 1.249 & 0.243 & 1 & 0.622 & 0.540 & 0.047 & 6.243 \\
Ascites & -0.863 & 0.994 & 0.755 & 1 & 0.385 & 0.422 & 0.060 & 2.957 \\
Residual tumor & 0.267 & 1.142 & 0.055 & 1 & 0.815 & 1.307 & 0.139 & 12.262 \\
Age & 0.047 & 0.034 & 1.951 & 1 & 0.162 & 1.049 & 0.981 & 1.121 \\
CTSB semi-quantitative & 1.640 & 0.803 & 4.165 & 1 & $\mathbf{0 . 0 4 1}$ & 5.155 & 1.067 & 24.896 \\
CC semi-quantitative & 0.658 & 0.338 & 3.780 & 1 & 0.052 & 1.931 & 0.995 & 3.749 \\
CTSL semi-quantitative & -0.208 & 0.295 & 0.497 & 1 & 0.481 & 0.812 & 0.456 & 1.448 \\
CTSD semi-quantitative & 0.630 & 0.495 & 1.624 & 1 & 0.202 & 1.878 & 0.713 & 4.950 \\
CTSB expression & -0.257 & 1.576 & 0.027 & 1 & 0.870 & 0.773 & 0.035 & 16.962 \\
CC expression & 0.211 & 1.199 & 0.031 & 1 & 0.860 & 1.235 & 0.118 & 12.959 \\
CTSL expression & 1.919 & 0.938 & 4.184 & 1 & $\mathbf{0 . 0 4 1}$ & 6.814 & 1.084 & 42.848 \\
CTSD expression & 0.412 & 1.032 & 0.159 & 1 & 0.690 & 1.510 & 0.200 & 11.409 \\
\hline
\end{tabular}

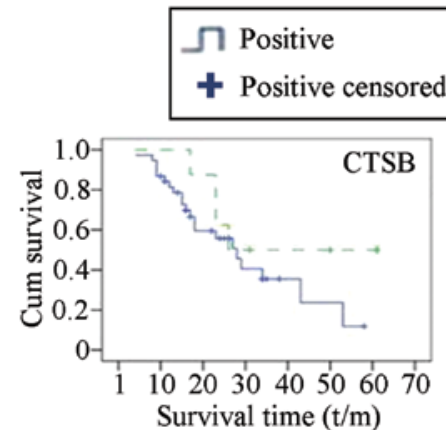

\section{Negative \\ Negative censored}
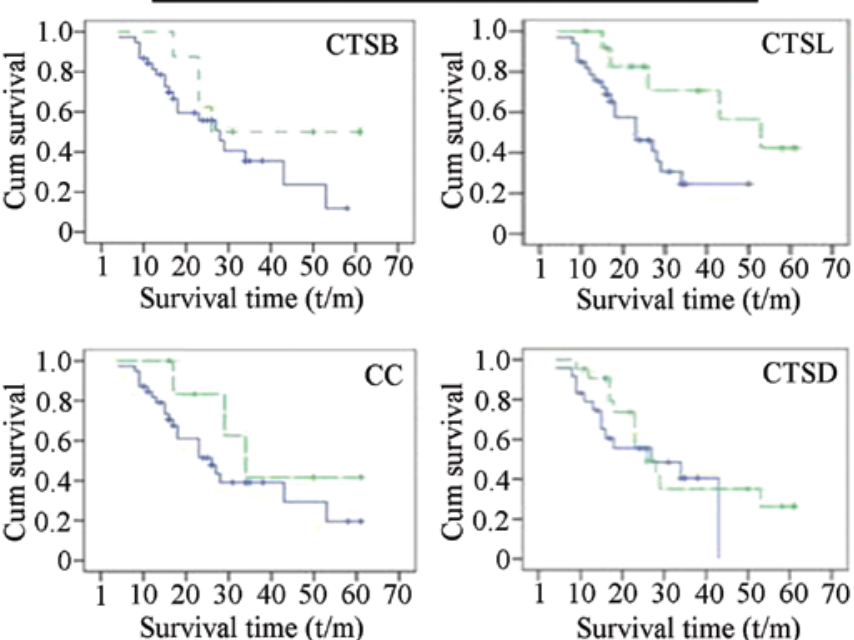

Figure 2. Survival curve of ovarian cancer patients with or without mRNA expression of CTSB, CTSL, CC and CTSD in tumors. A statistically significant difference in the survival time was noted between ovarian cancer patients with or without CTSL gene expression $(45.33 \pm 5.623$ vs. $26.64 \pm 2.955$ months, $\mathrm{P}<0.05)$. However, no statistically significant difference in survival time was observed between ovarian cancer patients with or without CTSB gene expression, CC expression and CTSD gene expression.

times of the patients with CTSL-positive and CTSL-negative tumors were $26.64 \pm 2.955$ and $45.33 \pm 5.623$ months, respectively, with statistically significant differences $(\mathrm{P}<0.05)$. The survival times of patients with upregulated and downregulated expression of $\mathrm{CC}$ were $31.83 \pm 3.649$ and $41.375 \pm 7.624$ months $(\mathrm{P}<0.05)$, respectively, and the survival times of patients with
Table IV. Comparison of the serum levels of CTSL in different ovarian tissues.

\begin{tabular}{lcc}
\hline Group & $\mathrm{n}$ & $\begin{array}{c}\text { Serum levels of CTSL } \\
{[\mu \mathrm{g} / 1,(\text { mean } \pm \mathrm{SD})]}\end{array}$ \\
\hline Healthy control group & 101 & $5.59 \pm 1.75^{\mathrm{a}}$ \\
Benign ovarian tumor group & 100 & $10.97 \pm 3.84^{\mathrm{b}}$ \\
Malignant ovarian tumor group & 177 & $21.59 \pm 8.24$ \\
\hline
\end{tabular}

${ }^{\mathrm{a}}$ Compared to normal control, $\mathrm{P}=0.000$; ${ }^{\mathrm{b}}$ compared to benign group and normal control, $\mathrm{P}=0.000$.

upregulated and downregulated expression of CTSD were $27.395 \pm 3.302$ and $34.462 \pm 4.617$ months $(\mathrm{P}<0.05)$, respectively.

To further study whether the expression levels of CTSB, CTSL, CC and CTSD are independent prognostic indicators for ovarian cancer, we performed the Cox regression model and multifactorial survival analysis to illustrate the relationship between prognosis and factors including age, histological type, histological grade, clinical stage, liver metastasis, omentum metastasis, lymph node metastasis, ascites, residual foci and semi-quantitative expression of CTSB, CTSL, CC and CTSD. As shown in Table III, expression levels of CTSB and CTSL were found to be independent prognostic factors for ovarian cancer.

Serum concentration of CTSL and its relationship with clinicopathological features, metastasis and prognosis in patients with malignant ovarian tumors. As shown in Table IV, the serum concentration of CTSL in patients with malignant ovarian tumors was significantly higher than the concentration 
Table V. Relationship between the CTSL levels in serum with clinicopathological variables in patients with ovarian cancers.

CTSL

Clinicopathologic factors

$\mathrm{n} \quad[\mu \mathrm{g} / \mathrm{l},($ mean $\pm \mathrm{SD})]$

Pathological type

Serous cystadenocarcinoma 109

Mucinous cystadenocarcinoma 54

$21.62 \pm 8.52$

Poorly differentiated

$20.28 \pm 7.44$

adenocarcinoma

$26.49 \pm 7.64$

Grade

I-II

29

$18.54 \pm 7.30$

III

148

$23.04 \pm 7.67$

FIGO stage

I-II

$19.66 \pm 7.83$

III-IV

115

$22.64 \pm 8.31$

Retroperitoneal lymph

node metastasis

$\begin{array}{lrc}\text { Positive } & 85 & 23.64 \pm 8.89 \\ \text { Negative } & 92 & 21.42 \pm 8.82 \\ \text { Pelvic metastases } & & \\ \text { Positive }^{\mathrm{b}} & 125 & 23.64 \pm 8.8 \\ \text { Negative } & 52 & 21.42 \pm 8.82 \\ \text { Peritoneal metastases } & & \\ \text { Positive }^{\mathrm{c}} & 115 & 22.96 \pm 8.41 \\ \text { Negative }^{\text {Distant metastasis }} & 62 & 19.07 \pm 7.36 \\ \text { Positive }^{\mathrm{d}} & & \\ \text { Negative }^{\text {Negati }} & 32 & 22.03 \pm 8.05 \\ \end{array}$

${ }^{\text {aAccording }}$ to the International Federation of Gynecology and Obstetrics (FIGO) surgical-pathologic stage (2004). ${ }^{\text {bLesions }}$ extended to the uterine fallopian tubes or other pelvic tissue; clesions extended to the pelvic complement organs such as the liver surface, spleen, small intestine, omentum or cross noon; desions extended to the lung, brain, bone or liver parenchyma.

levels in patients with benign ovarian tumors and in healthy controls $(\mathrm{P}=0.000)$. In addition, the level of CTSL in the benign group was notably higher than the level in the normal controls $(\mathrm{P}=0.000)$. The serum concentration of CTSL in ovarian cancer displayed no obvious differences among the pathological types. Likewise, the serum concentrations of CTSL in patients with lymphatic and pelvic metastasis, or with distant metastasis showed no significant differences when compared to the CTSL concentrations in patients without these metastases. However, the serum levels of CTSL in patients with low histological grade and advanced stage were higher than the levels in patients with high grade and early stage disease $(\mathrm{F}=12.452, \mathrm{P}=0.030)$, and the CTSL level in patients with peritoneal metastasis was higher than the level in patients without peritoneal metastasis $(\mathrm{F}=12.210, \mathrm{P}=0.030)$ (Table V).

The ROC curve was established based on the serum levels of CTSL in 177 patients with epithelial ovarian cancer (Fig. 3A). A comparison of the area under the curve between
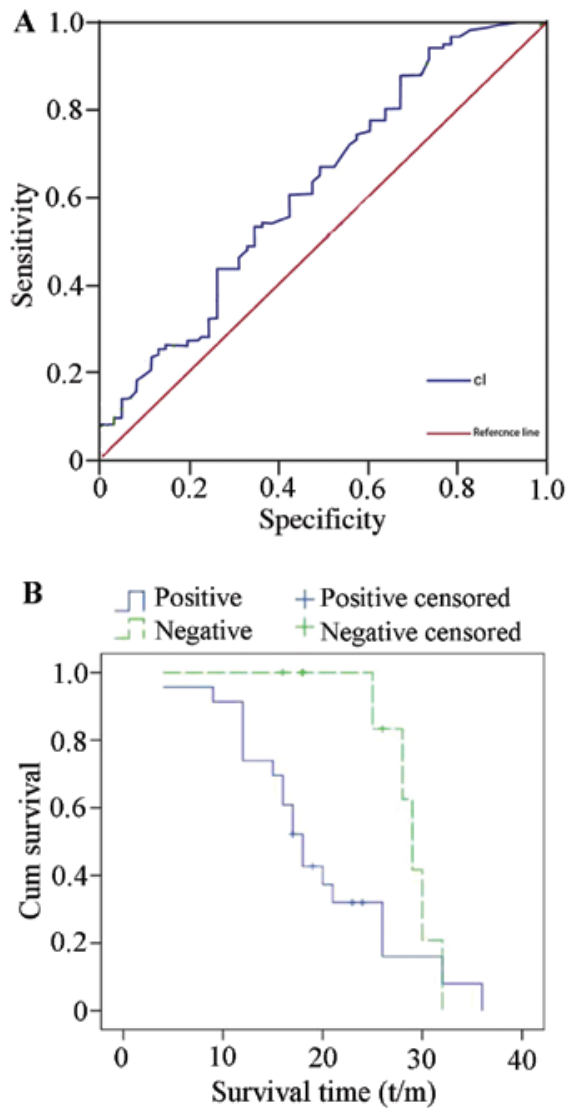

Figure 3. (A) ROC curve for determining metastases. (B) Kaplan-Meier curve for determining the survival of the ovarian cancer patients according to the expression of CTSL in serum.

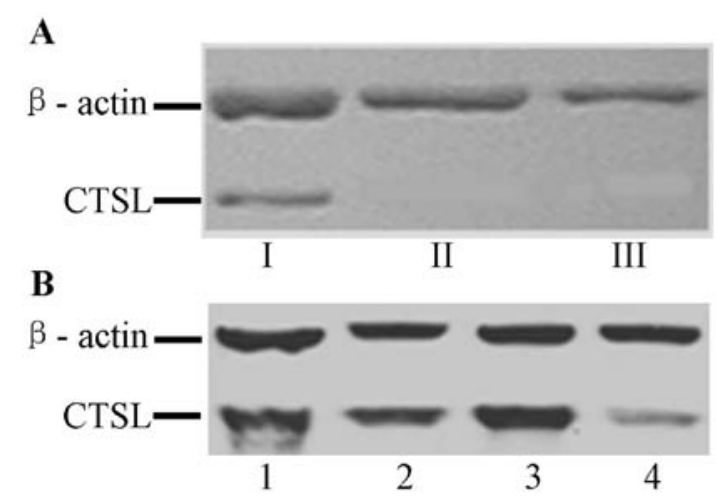

Figure 4. Protein expression of CTSL in ovarian cancer cells detected by western blotting. (A) The protein expression of CTSL in HO8910-CTSL cells; lane I, HO8910-CTSL cells; lane II, HO8910-pcDNA3.1 cells; lane III, HO8910 cells. (B) The CTSL protein expression in A2780 cells; lane 1, A2780 cells; lane 2, A2780-pSilencer cells; lane 3, A2780-Control cells; lane 4, A2780-CTSL cells.

115 patients with pelvic metastasis and 62 patients without metastasis was performed to estimate the sensitivity and specificity of serum CTSL levels. The results indicated that the area under the curve was 0.624 , and the sensitivity, specificity, positive likelihood ratio and negative likelihood ratio were $60.9 \%$ (70/115 cases), $57.4 \%$ (26/62 cases), 1.4 and 0.7 , respectively, suggesting that the serum CTSL levels may be potential markers for the preoperative assessment of tumor metastasis 
Table VI. Results of the Cox proportional hazards regression model analysis.

\begin{tabular}{lcccccccc}
\hline & & & & & & & \multicolumn{2}{c}{$95 \%$ CI } \\
\cline { 5 - 9 } Clinicopathological factors & B & SE & Wald & df & Sig & Exp(B) & Lower & Upper \\
\hline Tumor stage & 2.921 & 2.316 & 1.590 & 1 & 0.207 & 18.553 & 0.198 & $1,732.209$ \\
Tumor type & 12.132 & 7.922 & 2.345 & 1 & 0.126 & $185,779.3$ & 0.034 & $1 \mathrm{E}+012$ \\
Tumor grade & -0.878 & 1.076 & 0.666 & 1 & 0.414 & 0.416 & 0.050 & 3.424 \\
Liver metastasis & -3.197 & 1.723 & 3.444 & 1 & 0.063 & 0.041 & 0.001 & 1.197 \\
Omentum metastasis & 5.352 & 3.655 & 2.144 & 1 & 0.143 & 211.16 & 0.163 & $272,884.3$ \\
Lymph node metastasis & 3.233 & 2.084 & 2.407 & 1 & 0.121 & 25.368 & 0.427 & $1,507.240$ \\
Ascites & -1.103 & 1.589 & 0.482 & 1 & 0.488 & 0.332 & 0.015 & 7.474 \\
Residual tumor & 3.752 & 1.812 & 4.291 & 1 & 0.038 & 42.619 & 1.224 & $1,484.467$ \\
Serum concentration of CTSL & 0.056 & 0.059 & 0.920 & 1 & 0.337 & 1.058 & 0.943 & 1.186 \\
\hline
\end{tabular}

Table VII. Comparison of cell invasive, metastatic and adhesion abilities of ovarian cancer cells in vitro (mean \pm SD).

\begin{tabular}{lcccccccr}
\hline & \multicolumn{2}{c}{ Invasive ability } & & \multicolumn{2}{c}{ Metastatic ability } & & \multicolumn{2}{c}{ Adhesion ability } \\
\cline { 2 - 3 } Cell groups & Absorbance values & P-value & & Absorbance values & P-value & & Absorbance values & P-value \\
\hline HO8910 & $0.159 \pm 0.0468$ & & & $0.459 \pm 0.674$ & & & $0.156 \pm 0.035$ & \\
HO8910-pcDNA3.1 & $0.165 \pm 0.040$ & $>0.05^{\mathrm{a}}$ & & $0.486 \pm 0.027$ & $0.687^{\mathrm{a}}$ & & $0.193 \pm 0.041$ & $>0.05^{\mathrm{a}}$ \\
HO8910-CTSL & $0.343 \pm 0.178$ & $<0.05^{\mathrm{a}, \mathrm{b}}$ & & $1.252 \pm 0.114$ & $0.000^{\mathrm{a}, \mathrm{b}}$ & & $0.186 \pm 0.032$ & $>0.05^{\mathrm{b}}$ \\
A2780 & $0.4354 \pm 0.049$ & & & $0.2273 \pm 0.0746$ & & & $0.2023 \pm 0.080$ & \\
A2780-pSilencer & $0.4370 \pm 0.056$ & $0.970^{\mathrm{c}}$ & & $0.1776 \pm 0.0353$ & $0.095^{\mathrm{c}}$ & & $0.2015 \pm 0.044$ & 0.969 \\
A2780-Control & $0.3871 \pm 0.040$ & $0.281^{\mathrm{c}}$ & & $0.2083 \pm 0.0552$ & $0.589^{\mathrm{c}}$ & & $0.2073 \pm 0.044$ & 0.816 \\
A2780-CTSL & $0.2849 \pm 0.057$ & $0.007^{\mathrm{c}}$ & & $0.1340 \pm 0.046$ & $0.004^{\mathrm{c}}$ & & $0.2015 \pm 0.040$ & 0.969 \\
\hline
\end{tabular}

${ }^{a}$ Compared with HO8910 cells; ${ }^{b}$ compared with HO8910-pcDNA3.1 cells; ${ }^{c}$ compared with A2780 cells.

in ovarian cancer. As shown in Fig. 3B, the Kaplan-Meier survival curve showed that the average overall survival of patients with CTSL-positive tumors was $19.67 \pm 1.86$ months, while the survival of the patients with CTSL-negative tumors was $29.9 \pm 1.06$ months, indicated a statistically significant difference $(\mathrm{P}=0.036)$ in cumulative survival rate.

Cox regression model and multifactorial survival analysis were used to determine whether the CTSL expression in preoperative ovarian cancer patients is an independent prognostic indicator. Among all the factors including age, histological type, histological grade, clinical stage, liver metastasis, omentum metastasis, lymph node metastasis, ascites, residual foci and the preoperative serous content of CTSL, postoperative residual tumor size was found to be an independent prognostic factor $(\mathrm{P}=0.038)$ (Table $\mathrm{VI})$, but the preoperative serum concentration of CTSL had a weaker association with prognosis $(\mathrm{P}=0.337)$.

Expression of CTSL in ovarian cancer cells and its influence on cell invasion, metastasis and cell adhesion. RT-PCR results indicated that CTSL mRNA was positively expressed in HO8910-CTSL cells, and negatively expressed in HO8910 and HO8910-pcDNA3.1 cells. The western blotting indi- cated that the CTSL protein was positively expressed in HO8910-CTSL cells but negatively expressed in HO8910 and HO8910-pcDNA3.1 cells (Fig. 4A). These results indicate that the expression of CTSL was consistent at both the mRNA and protein levels.

RT-PCR results showed that the mRNA expression of CTSL in the A2780 ovarian cancer cells transfected with the siRNA 1202 sequence was obviously lower than the expression in A2780 cells transfected with the other sequence or the control sequence $(\mathrm{P}<0.05)$. Thus, the fragment of 1202 sequence was selected to construct the siRNA interference eukaryotic expression vector of CTSL. RT-PCR and western blot results showed that the expression of CTSL at the mRNA and protein levels was downregulated in A2780-CTSL cells, but no significant difference in expression was observed among the A2780, A2780-Control and A2780-pSilencer cells, respectively (Fig. 4B).

The expression of CTSL had less influence on cell growth and proliferation in accordance with the cell growth curve and the cell colony formation assay in the HO8910-CTSL (+), HO8910-pcDNA3.1 and HO8910 cells, and A2780, A2780control and A2780-pSilencer cells, respectively. In addition, on the basis of FCM analysis, the percentages of cells in the 
S, G2 and M phases of the cell cycle in the HO8910-CTSL cell group were higher than these percentages in the HO8910pcDNA3.1 and HO8910 cells, and the percentages of cells in the S, G2 and M phases of the cell cycle in the A2780-CTSL (-) cell group were lower that these percentages in the A2780 cells and A2780-controls, although both showed no statistically significant differences. However, the expression of CTSL had obvious influences on cell invasion and metastasis. As shown in Table VII, the cell invasive and metastatic abilities of the HO8910-CTSL cells were notably increased when compared with these abilities in the control cells $(\mathrm{P}<0.05)$, and the abilities of the A2780-CTSL(-) cells were obviously decreased in comparison with the abilities of the control cells $(\mathrm{P}<0.05)$, while no changes were observed in the cell adhesion ability of HO8910-CTSL and A2780-CTSL(-) cells when compared with their corresponding controls.

\section{Discussion}

CTSs are a family of cysteine proteases which function primarily in protein degradation in the lysosomes of the majority of cell types (5), and specific CTSs are often upregulated in various types of cancers (10). CTSs are expressed at the cell surface of cancer cells and are secreted into the extracellular space, where they degrade ECM components $(11,12)$. This extracellular proteolytic activity allows cancer cells to invade surrounding tissue, blood and lymph vessels and to metastasize to tissues at distant sites (13). The present study aimed to explore the relationship between CTSB, CTSL, CC and CTSD mRNA expression in ovarian epithelial cancer and clinicopathological factors and prognosis. We observed that CTSB, CTSL and CC expression in malignant ovarian tumors was significantly higher than the expression levels in benign tumors and normal ovarian tissues, and CTSB was associated with the amount of ascites and histological type. CTSL was associated with clinical stage, histological grade and lymph node metastasis, and CC was associated with pathological grade, liver metastasis and omentum metastasis. In addition, the univariate survival analysis showed that CTSL expression was associated with patient prognosis, and COX analysis indicated that CTSB and CTSL expression was an independent prognostic factor in ovarian cancer.

Among all of the CTSs genes, CTSB and CTSL have been investigated the most intensively and appear to play a role in cancer based on their increased expression in various human cancers (14-16). A role of CTSB and CTSL in tumor cell invasion was suggested by the observation of the increased invasiveness of cells overexpressing CTSB and CTSL (17) and by the decreased invasion in the presence of specific inhibitors of CTSB and CTSL (18). Moreover, immunohistochemical analysis demonstrated that CTSB and CTSL exist in the cytoplasm of tumor cells in human ovarian cancer $(9,19)$. Similarly, in the present study increased expression of CTSB and CTSL was noted in cancer, but not normal ovarian tissue, suggesting that CTSB and CTSL are survival prognostic factors in ovarian cancer, and may contribute to the invasiveness of ovarian cancer cells. Regarding their mechanism of action, previous studies indicate that they play a catalytic role (20-22). First, CTSB and CTSL can act as protease, directly or indirectly, degrading the catalytic extracellular matrix, so that the physical barrier around the tumor cells is destroyed. Secondly, the intercellular adhesion is remodeled, so that the tumor cells grow into the surrounding area. Third, they act on the matrix components to promote the biological activity of tumor cells; and fourth, tumor neovascularization is promoted, directly or indirectly, to promote vascular endothelial cell sprouting and invasive growth.

Since ovarian cancer tissue is highly heterogeneous, multiple biopsies are necessary for careful examination $(23,24)$. This means that the quantitation of cathepsins in biological fluids from ovarian cancer patients has several clinical advantages over measurements from ovarian cancer tissue. We found that the serum levels of CTSL were significantly higher in patients with ovarian malignant tumors than these levels in benign tumors and healthy controls, and the CTSL levels were elevated in low grade and advanced stage disease when compared to the levels in high grade and early stage disease. Our results were consistent with previous research (7). Siewinski et al (25) reported that the serum level of CTSL was higher in malignant tumors than that in benign tumors and normal controls. Women with ovarian cancer were found to have higher levels of CTSB and CTSL in sera (26), and CTSB and CTSL were present in ascites and cyst fluid of patients with ovarian cancer $(15,27)$. These results indicate that serum CTSL is increased in patients with ovarian cancer, and it may be a valuable serum markers for the diagnosis of ovarian cancer. Due to the occult nature of ovarian cancer onset, during early diagnosis and preoperative diagnosis it is difficult to judge the degree of invasion and metastasis resulting in the difficulty in treatment decision making and implementation. Based on the fact that the CTSL content in the peripheral blood of ovarian cancer patients was found to be related to invasion and metastasis, it is worth investigating whether it can be used as a marker before surgery to determine the extent of tumor invasion and metastasis. Observations in this group suggest that the CTSL content in the peripheral blood of ovarian cancer patients was positively correlated with the degree of extrapelvic invasion and metastasis. The ROC and performance analysis of the degree of invasion and metastasis further indicated that there was clinical reference value to determine the degree of tumor invasion and metastasis. Diagnostic and differential diagnoses of ovarian cancer pelvic metastasis rely mainly on imaging techniques. Research has confirmed that for ultrasound, calculate scan imaging (CT) or magnetic resonance imaging (MRI) examination in the peritoneum, mesentery, omentum, lesions $<2 \mathrm{~cm}$ in diameter are difficult to identify. In regards to other diseases such as chronic inflammation or proliferationresistant tuberculosis, the mass identification and performance were similar to ovarian cancer, for both the clinical misdiagnosis rate was up to $30 \%$ (28). The peripheral blood CTSL concentration was associated with malignant cell degradation in the matrix, rather than inflammatory lesions. Therefore, determination of the CTSL content in peripheral blood could be used as a reference marker to assess the degree of tumor invasion and metastasis, especially to ascertain whether there is an extrapelvic metastasis prior to surgery.

Cell adhesion, invasive and migratory abilities are important for tumor cell invasion and metastasis. Our results showed that the invasive and migratory abilities of pcDNA3.1CTSL(+)-HO8910 cells were significantly greater than the 
abilities of the control cells in vitro, suggesting that the CTSL gene may play important roles in invasion and metastasis of ovarian cancer cells by hydrolysis of the basement membrane. Studies have shown that CTSL gene knockout mice exhibit a decline in tumor cell invasiveness. Levicar et al (29) found that CTSL is a protein which can modify the degree of malignancy of glioblastoma. In addition, we found that the cell invasive and migratory abilities of A2780 cells were decreased significantly while the CTSL expression in A2780 cells was downregulated by siRNA, providing further evidence that CTSL expression in tumor cells contributes to the invasion and migration of ovarian cancer cells, and this result is consistent with the findings of Yang and Cox (30) who reported that the downregulation of CTSL expression in melanoma cells reduced the ability of tumor cell invasion and metastasis, but had no influence on cell adhesion. Similar results in human glioma IPTP24 cells were reported by Levicar et al (29).

Taken together, on the basis of our findings in ovarian cancer and the related studies in other types of cancers, we conclude that the CTSL gene is involved in tumor invasion and metastasis through degradation of the extracellular matrix, without affecting the adhesion of ovarian cancer cells. Thus, the CTSL gene is a possible molecular target for blocking ovarian cancer invasion and metastasis.

\section{Acknowledgements}

The present study was supported by a grant from the Provincial Research Project Funding of Guangxi, China (no. 2010GXNSFD013053).

\section{References}

1. Jemal A, Thomas A, Murray T and Thun M: Cancer statistics, 2002. CA Cancer J Clin 52: 23-47, 2002.

2. Murdoch WJ, Van Kirk EA and Alexander BM: DNA damages in ovarian surface epithelial cells of ovulatory hens. Exp Biol Med (Maywood) 230: 429-433, 2005.

3. Deraco M, Baratti D, Laterza B, Balestra MR, Mingrone E, Macri A, Virzi S, Puccio F, Ravenda PS and Kusamura S: Advanced cytoreduction as surgical standard of care and hyperthermic intraperitoneal chemotherapy as promising treatment in epithelial ovarian cancer. Eur J Surg Oncol 37: 4-9, 2011.

4. Johnatty SE, Beesley J, Paul J, Fereday S, Spurdle AB, Webb PM Byth K, Marsh S, McLeod H, Harnett PR, Brown R, DeFazio A and Chenevix-Trench G: $A B C B 1$ (MDR 1) polymorphisms and progression-free survival among women with ovarian cancer following paclitaxel/carboplatin chemotherapy. Clin Cancer Res 14: 5594-5601, 2008.

5. Turk V, Turk B and Turk D: Lysosomal cysteine proteases: facts and opportunities. EMBO J 20: 4629-4633, 2001.

6. Athanassiadou P, Sakellariou V, Petrakakou E, Athanassiades P, Zerva C, Liossi A and Michalas S: Cathepsin D immunoreactivity in ovarian cancer: correlation with prognostic factors. Pathol Oncol Res 4: 103-107, 1998.

7. Nishida Y, Kohno K, Kawamata T, Morimitsu K, Kuwano M and Miyakawa I: Increased cathepsin L levels in serum in some patients with ovarian cancer: comparison with CA125 and CA72-4. Gynecol Oncol 56: 357-361, 1995.

8. Kolwijck E, Kos J, Obermajer N, Span PN, Thomas CM, Massuger LF and Sweep FC: The balance between extracellular cathepsins and cystatin $\mathrm{C}$ is of importance for ovarian cancer. Eur J Clin Invest 40: 591-599, 2010.
9. Nishikawa H, Ozaki Y, Nakanishi T, Blomgren K, Tada T, Arakawa A and Suzumori K: The role of cathepsin B and cystatin $\mathrm{C}$ in the mechanisms of invasion by ovarian cancer. Gynecol Oncol 92: 881-886, 2004.

10. Jedeszko $C$ and Sloane BF: Cysteine cathepsins in human cancer. Biol Chem 385: 1017-1027, 2004.

11. Gocheva V and Joyce JA: Cysteine cathepsins and the cutting edge of cancer invasion. Cell Cycle 6: 60-64, 2007.

12. Mohamed MM and Sloane BF: Cysteine cathepsins: multifunctional enzymes in cancer. Nat Rev Cancer 6: 764-775, 2006.

13. Lankelma JM, Voorend DM, Barwari T, Koetsveld J, Van der Spek AH, De Porto AP, Van Rooijen G and Van Noorden CJ: Cathepsin L, target in cancer treatment? Life Sci 86: 225-233, 2010.

14. Yan S, Sameni M and Sloane BF: Cathepsin B and human tumor progression. Biol Chem 379: 113-123, 1998.

15. Lah TT and Kos J: Cysteine proteinases in cancer progression and their clinical relevance for prognosis. Biol Chem 379: 125-130, 1998.

16. Rao JS: Molecular mechanisms of glioma invasiveness: the role of proteases. Nat Rev Cancer 3: 489-501, 2003.

17. Szpaderska AM and Frankfater A: An intracellular form of cathepsin B contributes to invasiveness in cancer. Cancer Res 61: 3493-3500, 2001.

18. Premzl A, Zavasnik-Bergant V, Turk V and Kos J: Intracellular and extracellular cathepsin B facilitate invasion of MCF-10A neoT cells through reconstituted extracellular matrix in vitro. Exp Cell Res 283: 206-214, 2003.

19. Scorilas A, Fotiou S, Tsiambas E, Yotis J, Kotsiandri F, Sameni M, Sloane BF and Talieri M: Determination of cathepsin B expression may offer additional prognostic information for ovarian cancer patients. Biol Chem 383: 1297-1303, 2002.

20. Wang M, Tang J, Liu S, Yoshida D and Teramoto A: Expression of cathepsin B and microvascular density increases with higher grade of astrocytomas. J Neurooncol 71: 3-7, 2005.

21. He X, Brenchley PE, Jayson GC, Hampson L, Davies J and Hampson IN: Hypoxia increases heparanase-dependent tumor cell invasion, which can be inhibited by antiheparanase antibodies. Cancer Res 64: 3928-3933, 2004.

22. Ricciardelli $C$ and Rodgers RJ: Extracellular matrix of ovarian tumors. Semin Reprod Med 24: 270-282, 2006.

23. Hogdall EV, Christensen L, Hogdall CK, Blaakaer J, Gayther S, Jacobs IJ, Christensen IJ and Kjaer SK: Prognostic value of estrogen receptor and progesterone receptor tumor expression in Danish ovarian cancer patients: from the 'MALOVA' ovarian cancer study. Oncol Rep 18: 1051-1059, 2007.

24. Falcetta F, Lupi M, Colombo V and Ubezio P: Dynamic rendering of the heterogeneous cell response to anticancer treatments. PLoS Comput Biol 9: e1003293, 2013.

25. Siewinski M, Saleh Y, Gryboc M, Murawski M, Ekonjo GB, Ziolkowski P, Janocha A and Symonowicz K: Determination of cysteine peptidases-like activity and their inhibitors in the serum of patients with ovarian cancer treated by conventional chemotherapy and vitamin E. J Exp Ther Oncol 4: 189-193, 2004.

26. Warwas M, Haczynska H, Gerber J and Nowak M: Cathepsin B-like activity as a serum tumour marker in ovarian carcinoma. Eur J Clin Chem Clin Biochem 35: 301-304, 1997.

27. Kolwijck E, Massuger LF, Thomas CM, Span PN, Krasovec M, Kos J and Sweep FC: Cathepsins B, L and cystatin C in cyst fluid of ovarian tumors. J Cancer Res Clin Oncol 136: 771-778, 2010.

28. Epelman M, Chikwava KR, Chauvin N and Servaes S: Imaging of pediatric ovarian neoplasms. Pediatr Radiol 41: 1085-1099, 2011.

29. Levicar N, Dewey RA, Daley E, Bates TE, Davies D, Kos J, Pilkington GJ and Lah TT: Selective suppression of cathepsin L by antisense cDNA impairs human brain tumor cell invasion in vitro and promotes apoptosis. Cancer Gene Ther 10: 141-151, 2003.

30. Yang Z and Cox JL: Cathepsin L increases invasion and migration of B16 melanoma. Cancer Cell Int 7: 8, 2007. 